\title{
NMR/Binomial Theorem Determination of the Number and the Proportions of Isomers of Poly(identical) Spaced Functional Group Substrate Reactions
}

\author{
Nejib Hussein Mekni ${ }^{1,2}$ \\ ${ }^{1}$ Department of Chemistry, Faculty of Sciences, Taibah University, Al-Munawarah, Saudi Arabia \\ ${ }^{2}$ Laboratory of Structural Organic Chemistry, Department of Chemistry, Faculty of Science of Tunis, El Manar \\ University, 2092 Tunis, Tunisia \\ Correspondence: Nejib Hussein Mekni, Laboratory of Structural Organic Chemistry, Department of Chemistry, Faculty \\ of Science of Tunis, El Manar University, 2092 Tunis, Tunisia. E-mail: n.mekni@gmail.com
}

Received: March 23, 2017 Accepted: April 18, 2017 Online Published: April 18, 2017

doi:10.5539/ijc.v9n2p72

URL: https://doi.org/10.5539/ijc.v9n2p72

\begin{abstract}
${ }^{1} \mathrm{H},{ }^{19} \mathrm{~F}$ or ${ }^{31} \mathrm{P}$ NMR integration data of the specific signals of the reaction products of the multi(identical) spaced functional group reactants, followed by the binomial theorem calculation allows to the same results obtained from GC analysis. According to the unsymmetrical or symmetrical parts of the substrate structure, the number of products may be determined.
\end{abstract}

Keywords: percent composition, organic mixture, ${ }^{1} \mathrm{H},{ }^{19} \mathrm{~F},{ }^{31} \mathrm{P}$ NMR titration, binomial theorem, multi(identical) functional group

\section{Introduction}

Compounds containing multi(identical) spaced functional groups exist in some natural substances (Hausmann et $a$. 2006) and synthesized products (Barton et al., 2016). In polymer field bis(functional) compounds are well described, they are used as monomers (Qomi et $a l ., 2007$ ), but in major cases these compounds are not so far from to each other and they undergo chain polymerization reactions.

Some functional groups undergo competitive reactions, yielding isomers such as Markovnicov/anti-Markovnicov products of ionic addition on alkenes (Lorusso et al., 2006) and alkynes (Trostyanskaya et al., 2012), Z/E elimination reaction products (Darcsi et al., 2017; Bhalla et al., 2016), nucleophilic oxirane ring opening reaction products (Larin et al., 2014; Marié et al. 2007) etc.

The determination of the number and the proportions of the reaction products in the mixture is not evident from NMR data only, in this kind of reaction products is generally confirmed by gas chromatography (GC) data (Mekni et al., 2002).

Herein we focused on the determination of the number of products and their proportions from NMR data, followed by the binomial theorem calculations and their comparison to those obtained from the GC data. Our idea is consolidated by some experimental results previously published.

\section{Results and Discussion}

$n$ identical spaced functional groups molecule containing, are considered to react simultaneously as well as independent molecules. Each reactional functional group can yields to two isomer products (via $a$ or $b$ possibilities, omitting the stereochemistry) (Schemes 1,2). The reactions on each side are simultaneous and the same but with different probabilities on $a$ and $b$ routes).

The ${ }^{1} \mathrm{H},{ }^{19} \mathrm{~F}$ or ${ }^{31} \mathrm{P}$ nucleus NMR signals are integrable. But, the integration data can give information only about the $a$ and $b$ ratio. This information is unable to determine directly the number of products and the proportion of each component in the mixture, in both symmetrical and unsymmetrical parts of reactant molecules.

Starting from the simple case, by studding the bis(identical) spaced functional groups. If the two molecule parts are different (Scheme 1), the reaction yields $2^{2}=4$ products and the corresponding NMR spectra shows 4 signals $(2$ for $a$ and 2 for $b$ possibilities). 


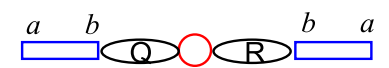

Scheme 1. Bis(identical) spaced functional groups.

The application of a probability calculation allows the determination of the proportion in each product (Equation 1).

$$
(a+b)^{2}=a^{2}+a b+b a+b^{2}
$$

If the molecule is symmetrical, the number of products decreases to 3 ( $a b$ is identical to $b a$ ). The number of NMR signals is also equal to 2 for each part ( 2 signals for $a$ and 2 signals for $b$ possibilities). The proportion of the three products is determined from the NMR signals integration data applied to the binomial theorem, in which the mixed product is the sum of the cross reactions $(a b+b a)$ :

$$
(a+b)^{2}=a^{2}+2 a b+b^{2}
$$

There are some experimental results of our previous works, justifying this later calculation for elimination (Mekni et $a l$., 2002; Mekni, 2014) and addition (Mekni et al., 2000) reactions (Table I). The integration of ${ }^{1} \mathrm{H}$ or ${ }^{19} \mathrm{~F}$ NMR signals followed by the application of the binomial theorem allows the determination of the number of products and their proportions. The obtained results are in total correlation with the GC data (Table 1).

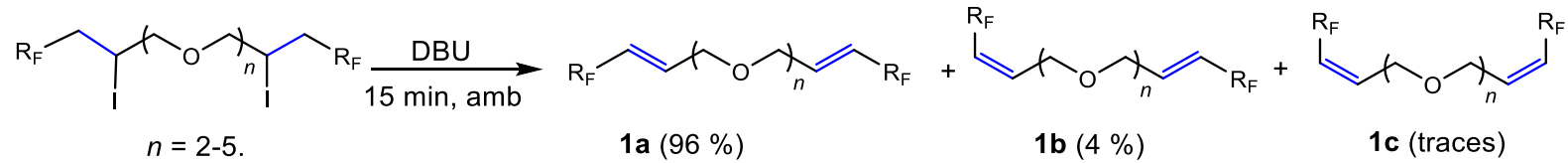

Scheme 2. Example of elimination reaction on bis(iodo) spaced functional groups.

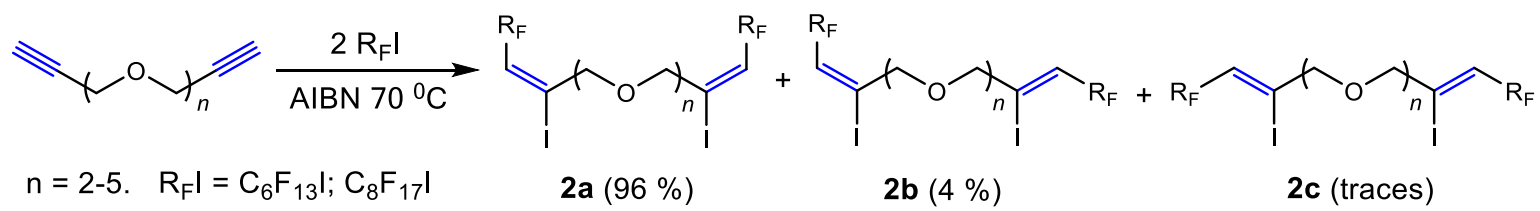

Scheme 3. Example of radical addition reaction on bis(alkyne) spaced functional groups.

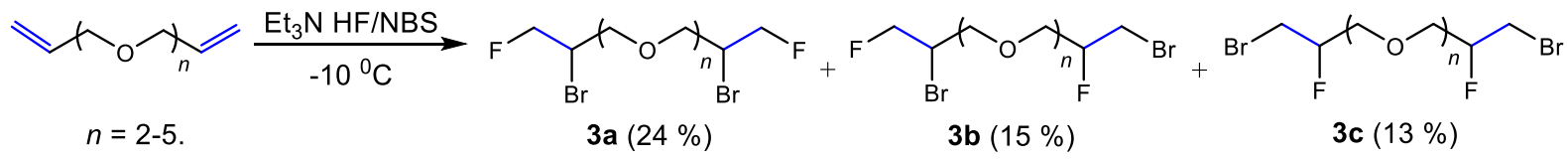

Scheme 4. Example of ionic addition reaction on bis(alkene) spaced functional groups

The results of the latter reaction (Scheme 4) are not well significant in term of the proportions because there are simultaneous degradation reactions (Mekni et al., 2000). But, they confirm clearly the formation of the mixed product theoretically expected product.

Table 1. Examples of bis(identical) spaced functional group substrate reactions.

\begin{tabular}{cccccl}
\hline Products & ${ }^{1}$ H NMR $(a / b)$ & ${ }^{19}$ F NMR $(a / b)$ & NMR $(\%)$ & GC $(\%)$ & \multicolumn{1}{c}{ Reference } \\
\hline $\mathbf{1 a} / \mathbf{1 b} / \mathbf{1 c}$ & $98 / 2$ & $98 / 2$ & $96 / 4 / 0$ & $96 / 4 / 0$ & Mekni et $a l .$, 2002 \\
$\mathbf{2 a / 2 b} / \mathbf{2 c}$ & $66 / 34$ & $66 / 34$ & $44 / 45 / 11$ & $44 / 45 / 11$ & Mekni, 2014 \\
$\mathbf{1 a} / \mathbf{1 b} / \mathbf{1} \mathbf{c}^{*}$ & $62 / 38$ & $61 / 39$ & $25 / 15 / 12$ & $24 / 15 / 13$ & Mekni et al., 2000 \\
\hline
\end{tabular}

If the molecule contains three different parts containing identical spaced reactional functional groups, it yields $2^{2}=8$ products for which the proportions are given according to the binomial equation 2 .

$$
(a+b)^{3}=a a a+a a b+a b a+a b b+b a a+b b a+b a b+b b b
$$




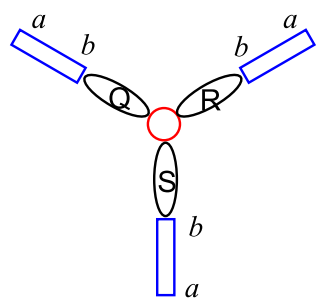

Scheme 5. Tris(identical) spaced functional groups.

If the substrate molecule parts are identical, the number of products becomes equal to 4 ( $a b$ is identical to $b a$ ). The calculation in this case follows the relation:

$$
(a+b)^{3}=a^{3}+3 a^{2} b+3 a b^{2}+b^{3}
$$

In the case of a molecule containing four (identical) reactional functional groups, linked to different parts, the number of products will be $2^{4}=16$, according to the binomial theorem (Equation 3 ):

$$
\begin{array}{ccc}
(a+b)^{4}=a a a a & +a b a a+a a b a+a a a b+b a a a+b b a a+b a b a+b a a b+a a b b & +a b a b+a b b a+b b b a+b a b b \\
& +b b a b+a b b b+b b b b
\end{array}
$$

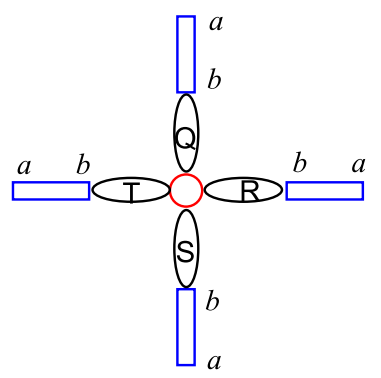

Scheme 6. Tetra(identical) spaced functional groups.

If the molecule parts are identical, the number of isomers decreases to 5 .

$$
a^{4}+4 a^{3} b+6 a^{2} b^{2}+4 a b^{3}+b^{4}
$$

The application of the bionomial theorem to the NMR integration data to calculate the number of isomers and their proportions in the mixture is true for the first, second order. So, by recurrence this calculation is applicable to the $n$ order in both cases symmetrical and unsymmetrical molecule substrate.

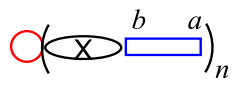

Scheme 7. Poly(identical) spaced functional groups.

The number of product is equal to $2^{n}$ and their proportions are given by the binomial theorem (Equation 4) applied to the corresponding NMR integration nucleus data.

$$
(a+b)^{n}=a^{n}+\left(C_{1}^{n}\right) a^{n-1} b+\left(C_{2}^{n}\right) a^{n-2} b^{2}+\ldots+\left(C_{n-1}^{n}\right) a b^{n-1}+b^{n}
$$

Particularly, if the parts of the molecule are identical, the number of isomers decreases to $n+1$ products, respecting Pascal's triangle in term of number of products and their proportions.

The obtained probability calculations results are in correlation with the chromatographic ones.

\section{Conclusion}

Because their signals are integrable, the ${ }^{1} \mathrm{H},{ }^{19} \mathrm{~F}$ or ${ }^{31} \mathrm{P}$ NMR data may be sufficient to determine the number of products and their proportions in a mixture of products resulting from multi(identical) functional group reagent. If the molecule parts are unsymmetrical, the number of products is given by the relation $2^{n}$, and decrease to $n+1$ isomers in the case of symmetrical reactants.

\section{References}

Barton, B., Hosten, E. C., \& Pohl, P. L. (2016). Discrimination between $o$-xylene, $m$-xylene $p$-xylene and ethylbenzene by host compound $(R, R)-(-)-2,3$-dimethyoxy-1,1,4,4-tetraphenylbutane-1,4-diol, Tetrahedron, 72, 8099-8105. 
https://doi.org/10.1016/j.tet.2016.10.062

Bhalla, A., Bari, S. S., Vats, S., Bhalla, J., Sharma, K., \& Narula, D. (2016). One pot, simple, and efficient synthesis of $(E)$ - and (Z)-3-allylidene- $\beta$-lactames from 3-allyl-3-phenylseleno- $\beta$-lactamase inhibitors, Tetrahedron Letters, 57 , 4763-4766. https://doi.org/10.1016/j.tetlet.2016.09.043

Darcsi, A., Racz, A., \& Beni, S. (2017). Identification and characterization of new dapoxetine impurty by NMR Tranaformation of $\mathrm{N}$-oxide by Cope elimination, Journal of Pharmaceutical and Biomedical Analysis, 134, 187-194. https://doi.org/10.1016/j.jpba.2016.11.029

Hausmann, R., Rettinger, J., Gerevich, Z., Meis, S., Kassack, M. U., Illes, P., Lambrecht, G., \& Schmalzing, G. (2006). The suramin analog 4,4',4",4"'-(carbonylbis(imino-5,1,3-benzenetriylbis (carbonylimino))) tetra-kisbenzenesulfonic acid (NF110) potently blocks P2X3 receptors: subtype selectivity is determined by location of sulfonic acid groups, Molecular Pharmacology, 6, 2058-2067. https://doi.org/10.1124/mol.106.022665

Larin, E. A., Kochubei, V. S., \& Atroshchenko, Y. M. (2014). Regio- and stereoselective synthesis of new diaminocyclopentanols, Beilstein Journal of Organic Chemistry, 10, 2513-2520. https://doi.org/10.3762/bjoc. 10.262

Lorusso, G., Di Masi, N. G., Maresca, L., Pacifico, C., \& Natile, G. (2006). Markovnikov versus anti-Markovnikov selectivity in the amination of terminal olefins coordinated to platinum(II), Inorganic Chemistry Communications, 9, 500-503. https://doi.org/10.1016/j.inoche.2006.01.014

Marié, J. C., Courillon, C., \& Malacria, M. (2007). Selective ring opening os silylated vinyloxiranes and reactivity of azido-alcohols, Arkivoc, 5, 277-292.

Mekni, N. H. (2014) Synthesis of new bis(3-perfluoroalkyl-1H-pyrazole) polyoxyethylene, Journal of Fluorine Chemistry, 168, 75-80. https://doi.org/10.1016/j.jfluchem.2014.09.008

Mekni, N., Hedhli, A., \& Baklouti, A. (2000). Bromofluorination of bis(allyl) polyoxyethylene ethers, Journal of Fluorine Chemistry, 101, 1-4. https://doi.org/10.1016/j.jfluchem.2014.09.008

Mekni, N., Hedhli, A., \& Baklouti, A. (2002). F-alkylation of bis(allyl) polyoxyethylene ethers, Journal of Fluorine Chemistry, 114, 43-46. https://doi.org/10.1016/S0022-1139(01)00554-1

Qomi, H. R., Habibi, A., \& Shahcheragh, S. M. (2017). Synthesis and fluorescence studies of nine 1,5-benzodiazepine-2,4-dione derivatives: Dual emission and excimer fluorescence, Spectrochimca Acta Part A: Molecular and Biomolecular Spectroscopy, 174, 164-170. https://doi.org/10.1016/j.saa.2016.11.028

Trostyanskaya, I. G., \& Beletskaya, I. P. (2012). Regio- and Stereoselective Copper-Catalyzed Addition of Aromatic and Aliphatic Thiols to Terminal and internal Nonactivated Alkynes, Synlett, 4, 535-540.

\section{Copyrights}

Copyright for this article is retained by the author(s), with first publication rights granted to the journal.

This is an open-access article distributed under the terms and conditions of the Creative Commons Attribution license (http://creativecommons.org/licenses/by/4.0/). 